\title{
A proposal of new operating procedure of transformer type fault current limiter
}

\section{AUTHOR(S):}

Shirai, Y; Taguchi, M; Shiotsu, M; Hatta, H; Muroya, S; Nitta, T

\section{CITATION:}

Shirai, Y...[et al]. A proposal of new operating procedure of transformer type fault current limiter. IEEE TRANSACTIONS ON APPLIED SUPERCONDUCTIVITY 2002, 12(1): 885-889

\section{ISSUE DATE:}

2002-03

URL:

http://hdl.handle.net/2433/50257

\section{RIGHT:}

(c)2002 IEEE. Personal use of this material is permitted. However, permission to reprint/republish this material for advertising or promotional purposes or for creating new collective works for resale or redistribution to servers or lists, or to reuse any copyrighted component of this work in other works must be obtained from the IEEE. 


\title{
A Proposal of New Operating Procedure of Transformer Type Fault Current Limiter
}

\author{
Y. Shirai, M. Taguchi, M. Shiotsu, H. Hatta, S. Muroya, and T. Nitta
}

\begin{abstract}
Recovery time of a superconducting fault current limiters (SCFCLs) of a transformer type is very short, because the fault current is limited by the inductance of the primary coil and the amount of energy dissipation in the secondary superconducting wire is small. The temperature of the secondary winding of the SCFCLs decreases even in the current limiting operation. It was pointed out that the SCFCL in the current limiting mode may recover to the waiting mode by shunting the current for a certain time. Recovery characteristics by FCL-short-circuit was studied experimentally. New operating procedure based on the recovery by FCL-short-circuit is proposed. Required FCL-short time for successful recovery was measured for various conditions. The availability of the proposed operating procedure was investigated using a one-machine infinite bus system with parallel transmission line including 3-phase SCFCL unit.
\end{abstract}

Index Terms-One machine infinite bus system, recovery characteristics, three-phase superconducting fault current limiter.

\section{INTRODUCTION}

$\mathbf{V}$ ARIOUS types of superconducting fault current limiters (SCFCLs) were proposed and have been studied, for example [1]-[4]. They are expected to improve reliability of power systems. An SCFCL of a transformer type with adjustable trigger current level was proposed and a trial single-phase one was designed and made [5]. The SCFCL consists of two LTC superconducting coils coupled co-axially. Inner (primary) coil will be connected to a power line. Outer (secondary) coil is short-circuited. Super/Normal transition at a fault occurs only in the secondary coil. The fault current is limited by the inductance of the primary coil. Therefore, the amount of energy dissipation in the secondary superconducting wire is small in the trial SCFCL. The recovery time (the required zero-current time for successful recovery from current limiting mode to waiting mode) is rather short, $450 \mathrm{~ms}$ at most. The basic test results showed that only a part of the secondary wire is in the normal state (normal zone) and the recovery time is shorter as the current limiting period is longer. That is, the temperature of the normal zone of the secondary wire decreases during the current limiting state [6].

Manuscript received September 24, 2001. This work was supported in part by the Japan Society for the Promotion of Science under Project JSPS-RFTF97P01004.

Y. Shirai, M. Taguchi, and M. Shiotsu are with the Department of Energy Science and Technology, Kyoto University, Yoshida-Honmachi, Sakyo-ku, Kyoto, 606-8501 Japan (e-mail: \{shirai; shiotsu\}@energy.kyoto-u.ac.jp taguchi@pe.energy.kyoto-u.ac.jp).

H. Hatta, S. Muroya, and T. Nitta are with Department of Electrical Engineering, the University of Tokyo, 7-3-1, Hongo, Bunkyo-ku, Tokyo, 113-8656 Japan (e-mail: \{hitta; muroya; nitta\} @ asc.t.u-tokyo.ac.jp).

Publisher Item Identifier S 1051-8223(02)03847-2.

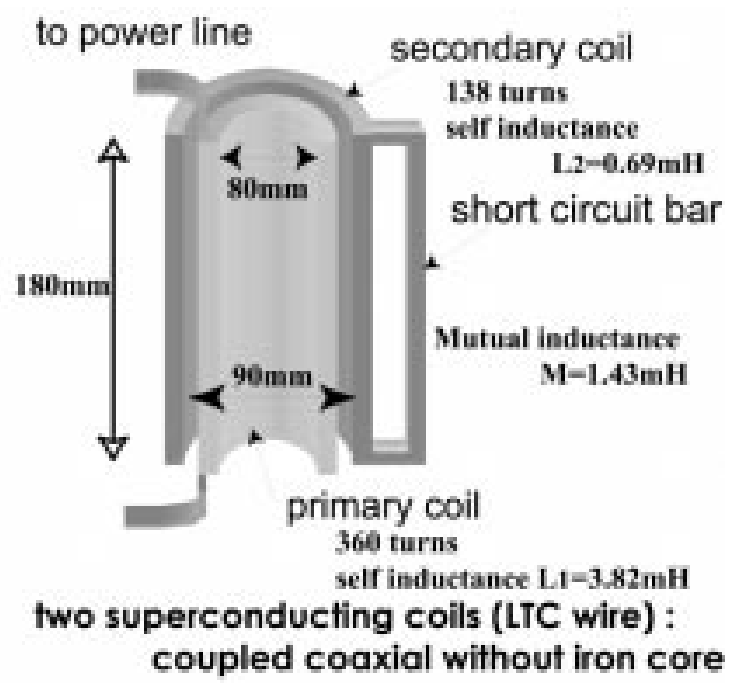

Fig. 1. Schematic configuration and specifications of proposed SCFCL (3 SCFCLs are in a cryostat).

In this paper, a new operating procedure of SCFCL using the above excellent feature was proposed and was studied experimentally. In general, after the current limiting operation starts, an SCFCL is isolated from the power line as soon as the fault current is shunted to the other impedance element, in order to suppress the temperature rise of the normal zone of the superconducting wire. However, the quick isolation from the power line is not necessary for the proposed SCFCL, because the temperature of the secondary wire decreases even in the current limiting mode. The proposed operating procedure is as follows. After the fault, the SCFCL continues the current limiting operation before the fault clear. After the fault clear, the SCFCL is short-circuited to recover to the waiting mode. The procedure contains no isolation process of SCFCL from the power line. The line current can flow continuously. The availability of the procedure was confirmed experimentally using a onemachine infinite bus system with parallel transmission line including 3-phase SCFCL unit.

\section{EXPERIMENT FOR RECOVERY BY FCL-SHORT CIRCUIT}

\section{A. Test SCFCL}

The test SCFCL unit for three-phase operation was designed and made [7]. It contains three SCFCLs of transformer type in one cryostat. The SCFCL consists of two superconducting coils (NbTi wire for AC use) coupled co-axially. The inner (primary) coil will be connected to a power line. The outer (secondary) coil is short-circuited. The primary coil can be slided with small 


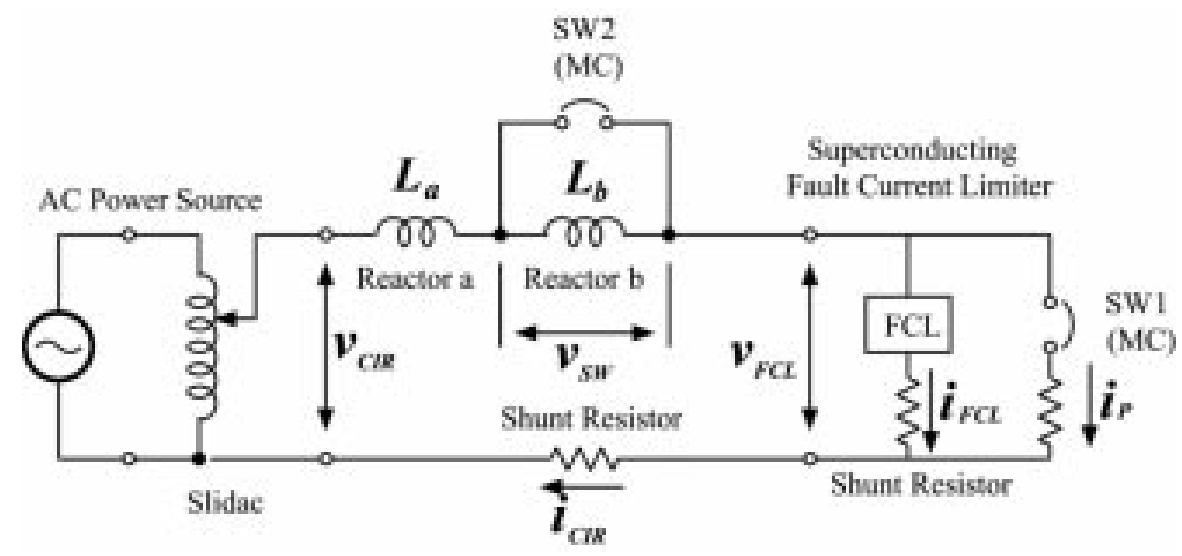

Fig. 2. Experimental circuit for recovery test by FCL-short circuit.

slide distance in order to calibrate the trigger current level [8]. The trigger current levels of three SCFCLs were adjusted to be the same value. In the waiting mode, both superconducting coils are in the superconducting state. When the fault current flowing through the SCFCL reaches the trigger current level, the super-normal transition occurs only at the secondary wire. The fault current is limited by the reactance of the primary coil. The designed trigger current level without slide distance of three SCFCLs is 85.7 Arms. Schematic configuration and specifications of the test SCFCL is shown in Fig. 1.

\section{B. Recovery Characteristics}

The recovery of an SCFCL from current limiting mode to the waiting mode is performed, in general, by isolation from a power line. The "Recovery Time" (the required zero current time of the SCFCL for recovery from the current limiting mode to the waiting mode) was measured for various "Fault Time" (the time while the fault current flows through the SCFCL) using test SCFCL [6]. It was confirmed that the "Recovery Time" depends on the "Fault Time." The "Recovery Time" decreases gradually and approaches to a certain value (a few ten ms), as the "Fault Time" is longer. The temperature of normal zone of the superconducting wire is expected to decrease, even if the fault current continues to flow through the SCFCL.

It was already reported [6] that, after a sufficient time from the fault occurrence, the current of the secondary winding becomes almost equal to the minimum propagation current of the superconducting wire. The joule heat loss per unit length at the normal zone balances with the heat flux at the surface of the normal zone of the wire (thermally equilibrium state). Therefore, the "Recovery Time" is quite short. It is an important advantage of this type SCFCL.

\section{Proposed Operating Procedure}

Because the temperature of the secondary wire decreases even in the current limiting mode, the SCFCL can continue to limit the fault current without burn out.

The proposed operating procedure is as follows:

1) At a fault, SCFCL turns into the current limiting mode immediately after the fault current reaches the trigger current level.
2) SCFCL continues to limit the fault current until the fault is clear.

3) After the fault clear, SCFCL is short-circuited so as to bypass the circuit current for a certain time and recovers to the waiting mode.

The procedure contains no isolation process of the SCFCL from the line and the line current flows continuously.

\section{Experimental Circuit for Recovery by Short Circuit}

In order to confirm the availability of the proposed operating procedure, the fundamental test using one of the three-phase SCFCLs was carried out with the experimental circuit shown in Fig. 2. Two reactors "a" and "b" and the SCFCL are connected in series to the AC power source $(50 \mathrm{~Hz})$ through a variable voltage transformer (slidac). A short-circuit switch SW2 (MC: Magnetic Controlled Contact) is connected in parallel to the reactor "b" to simulate a fault. The inductance $L_{a}$ and $L_{b}$ of the reactors are $2.13 \mathrm{mH}$ and $6.40 \mathrm{mH}$, respectively. A switch SW1 (MC) is used to shot-circuit the SCFCL in order to shunt the line current for recovery. The current $i_{F C L}$ of the SCFCL, the circuit current $i_{C I R}$, the current $i_{p}$ of the bypass circuit, the voltage $v_{F C L}$ of the SCFCL, the voltage $v_{C I R}$ of the output voltage of the slidac and the voltage $v_{S W}$ across the reactor "b" were measured.

\section{E. Test Procedure}

The switching sequence of SW1 and SW2 for recovery test by FCL-short-circuit is shown in Fig. 3. At the initial condition, SW1 and SW2 are open and the current flows through two reactors and SCFCL. SW2 is closed to simulate a fault. The SCFCL begin to limit the fault current. After a certain time, SW2 is opened to clear the fault. The SCFCL is still in the current limiting mode. Then the SCFCL is short-circuited by SW1 for a certain time to recover to the waiting mode.

\section{F. Experimental Results}

One of the experimental results is shown in Fig. 4. The voltage $v_{C I R}$ is $92 \mathrm{Vrms}$ and the circuit current $i_{F C L}$ is 32.9 Arms as initial conditions. The SCFCL begins to limit the fault current immediately after the fault occurred $(0.082 \mathrm{~s})$. The fault current is limited to be 49.5 Arms. It would be 93.6 


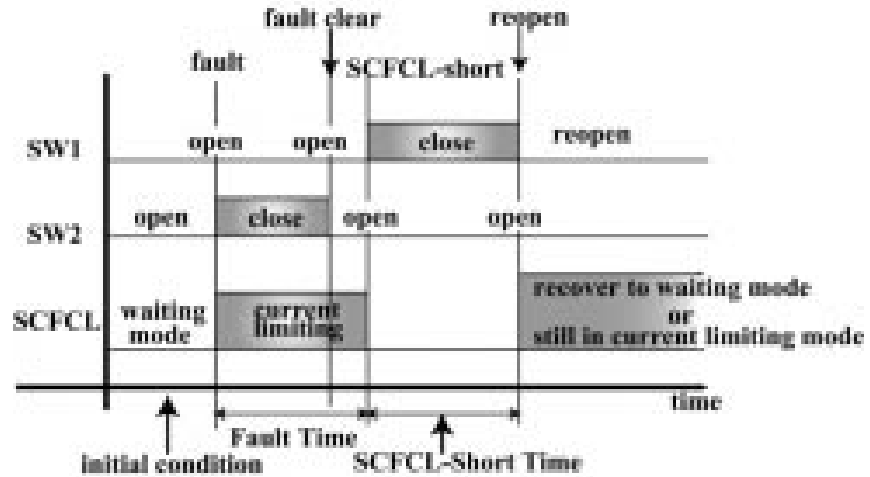

Fig. 3. Switching sequence of SW1 and SW2 for recovery test by FCL-short circuit.

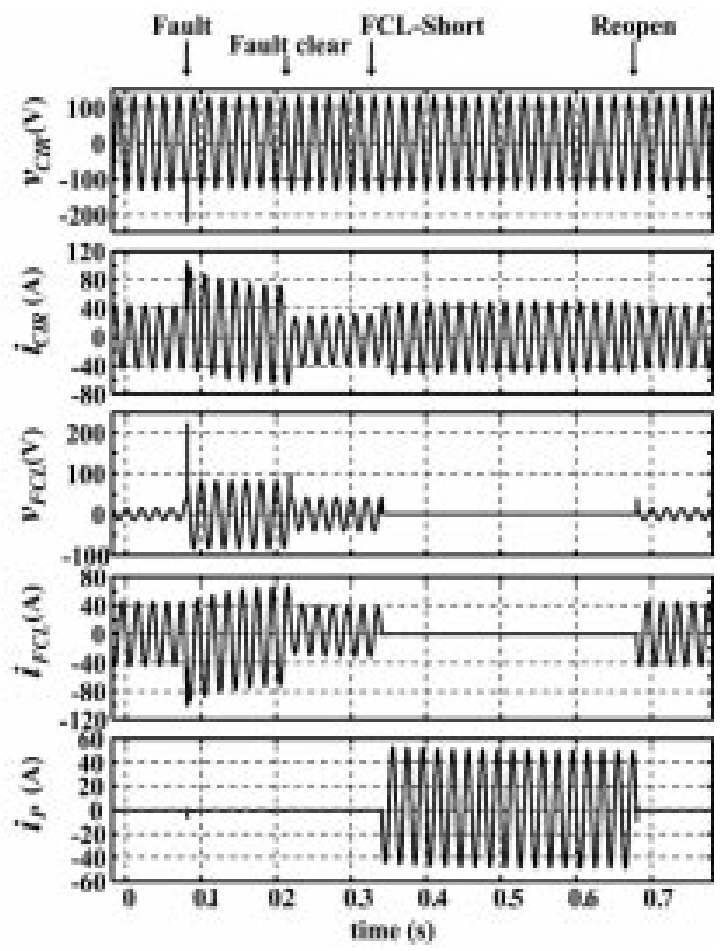

Fig. 4. Experimental result of current limiting operation with recovery by FCL-short-circuit.

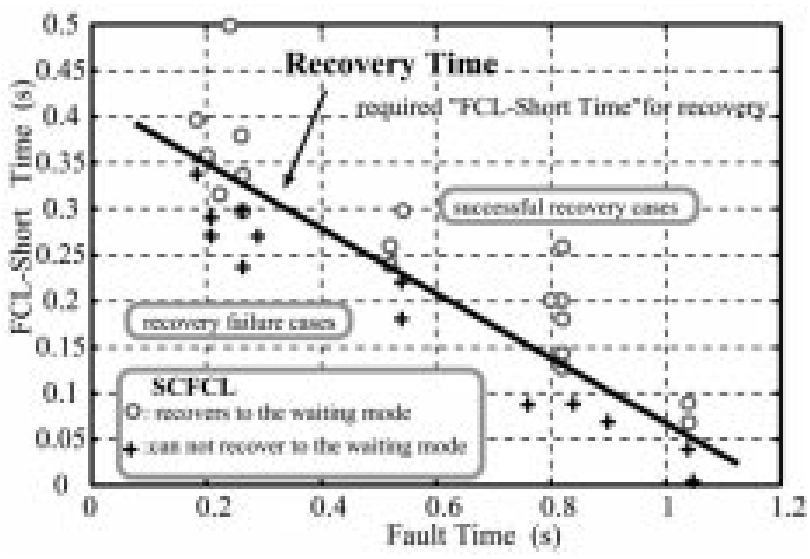

Fig. 5. Required "FCL-Short Time" for successful recovery versus "Fault Time."

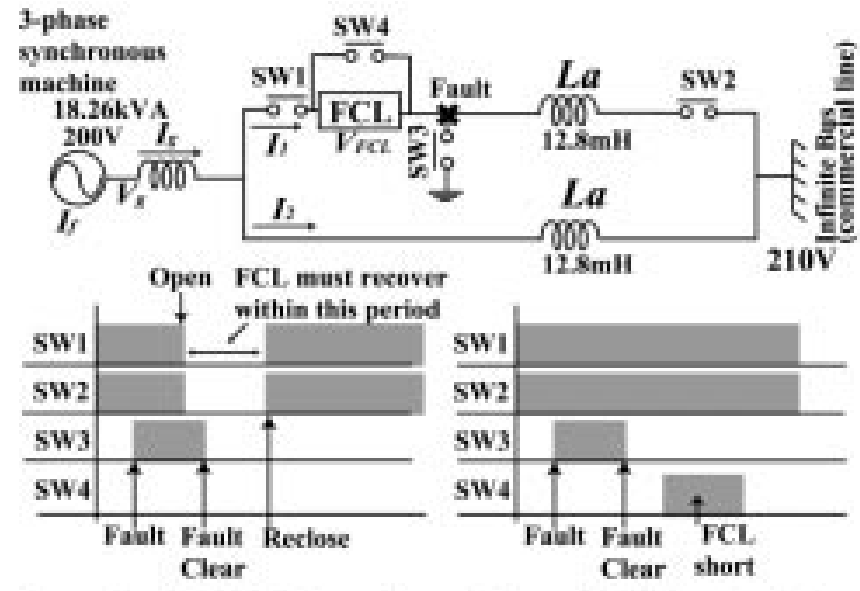

Conventional Switching Procedure

Fig. 6. One machine infinite bus system with SCFCL.

Arms without the SCFCL. The SW2 is opened at $0.218 \mathrm{~s}$ to clear the fault. The SCFCL is still in the current limiting mode and the circuit current is 24.7 Arms less than the initial current 32.9 Arms. The switch SW1 is closed to short-circuit the FCL at $0.343 \mathrm{~s}$. The interval time between the fault clear and the FCL-short is $125 \mathrm{~ms}$. The circuit current is commutated to flow through SW1 $\left(i_{p}\right)$ and the current through the SCFCL becomes less than a self-recovery current [6]. The recovery process starts. SW1 is re-opened at $0.681 \mathrm{~s}$ to the initial switching condition, and the SCFCL recovers to the waiting mode successfully in this experimental case. In this test case, "Fault Time" is $261 \mathrm{~ms}$ and "FCL-short Time" is $338 \mathrm{~ms}$. The circuit current $i_{C I R}$ flows continuously throughout the test operation.

\section{G. Required "FCL-Short Time" for Successful Recovery and "Fault Time"}

Fig. 5 shows the required "FCL-short Time" for successful recovery versus "Fault Time." The interval time between the fault clear and the beginning of FCL-short is $125 \mathrm{~ms}$ in all test cases. During this interval time, the temperature of the secondary wire may decrease.

The required "FCL-short Time" becomes smaller as the "Fault Time" is larger and saturated to about $50 \mathrm{~ms}$ for over $1 \mathrm{~s}$ of "Fault Time." The SCFCL can be short-circuited to recover at any time after the fault clear as far as the cooling system permits. The proposed operating procedure was performed successfully.

\section{CURREnt Limiting OPERATION IN ONE MACHINE INFINITE BUS SYSTEM}

\section{A. Experimental System}

Fig. 6 shows an experimental system and switching sequences of tests. A 3-phase synchronous generator is connected to an infinite bus ( $210 \mathrm{~V}$ commercial power line) through parallel artificial transmission lines. The upper line (fault line) has SW3 in parallel for simulated fault and has the three-phase SCFCL and circuit breakers (SW1 and SW2) in series. The ratings of the generator is $18.26 \mathrm{kVA}$ of capacity, $200 \mathrm{~V}$ of 


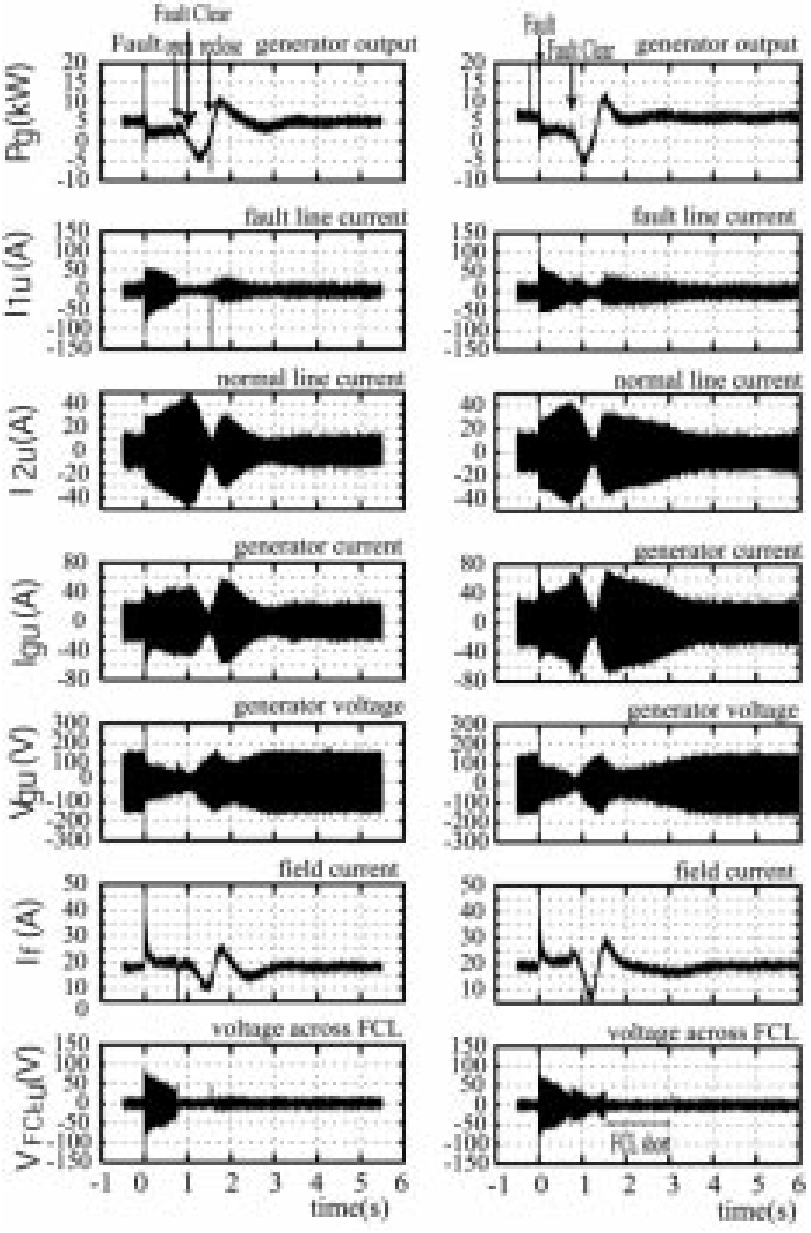

Fig. 7. Experimental result of current limiting operation in one-machine infinite bus system with the conventional switching sequence (left) and with the proposed operating procedure (right).

voltage, $52.5 \mathrm{~A}$ of current, $19.2 \mathrm{~A}$ of field current, $1500 \mathrm{rpm}$ of rotating speed, 4-poles, $50 \mathrm{~Hz}$. The field current is supplied by a constant voltage source. The switch SW3 is closed to simulate the 3-phase short fault. The switch SW4 connected in parallel to SCFCLs is closed to short-circuit the SCFCL for recovery. All switches are magnetically controlled and operated at the same time in three phases.

The generator current $I_{g}$, the voltage $V_{g}$, the field current $I_{f}$, the fault line current $I_{1}$, the normal line current $I_{2}$, the voltage $V_{F C L}$ across the FCL and the FCL current $I_{F C L}$ are measured.

\section{B. Proposed Operating Procedure}

In conventional switching sequence, the fault line including fault point and the SCFCL is isolated for a certain time by SW1 and SW2. The generator power flows through only one transmission line. The SCFCL should be recovered to the waiting mode within the isolation period. After a while, the fault line is re-closed back to the initial condition.

In the proposed operating procedure, the circuit breakers SW1 and SW2 do not work. After the fault clear, the SCFCL is short-circuited by SW4 for a certain time to recover. The fault line and the SCFCL are not isolated from the system.

\section{Experimental Results}

One of the experimental results with the conventional switching sequence is shown in Fig. 7(a). The generator output $P_{g}$ is $5 \mathrm{~kW}$ and the field current $I_{f}$ is 18 A for initial conditions. The wave forms of generator power, fault and normal line currents, and generator voltage, current, field current, and voltage across the SCFCL of u-phase are listed from top to bottom. After the 3-phase fault, the fault line currents of all phases reach the trigger current level $(125 \mathrm{~A})$ and all the SCFCLs turn into the current limiting mode. At the time of $0.77 \mathrm{~s}$, the fault line is isolated from the system by opening SW1 and SW2. The generator power is transmitted through the single line. This condition is severe for the stable operation. A quick re-closure is necessary. Then after the fault clear (SW3: open), SW1 and $\mathrm{SW} 2$ are re-closed at $1.55 \mathrm{~s}$. The large transient line current is observed at the re-closure. Small power swing is observed in $P_{g}$ after the re-closure.

Fig. 7(b) shows one of the experimental results with the proposed switching sequence. The generator output $P_{g}$ is $6 \mathrm{~kW}$ and the field current If is $19 \mathrm{~A}$ for initial conditions. At the time of $0.77 \mathrm{~s}$, the fault is cleared. The two lines are alive and the generator power can flow through the parallel lines. Therefore, a power for generator rotor acceleration is less than that in the conventional operation. The SCFCL is short-circuited from $1.55 \mathrm{~s}$ to $3.05 \mathrm{~s}$. All the SCFCLs recovered to the waiting mode successfully. The upper line (fault line) current continues to flow throughout the operation. The quick re-closure operation is not needed, but the SCFCL should be short-circuited after the fault is cleared. However, it is not necessary to short circuit the SCFCL quickly, because the "Recovery Time" becomes shorter as the "Fault Time" is longer. Any large transient line current at the SCFCL-short operation is not observed. It can be said that the proposed operating procedure is better for the transient stability of the system.

\section{CONCLUSION}

Recovery characteristics of the transformer type SCFCL by FCL-short-circuit was studied experimentally. It was confirmed that the SCFCL in the current limiting mode can recover to the waiting mode successfully by shunting the current for a certain time. Required FCL-short time for successful recovery was measured for various conditions. It becomes shorter, as the fault current limiting time is longer. New operating procedure based on the recovery by FCL-short-circuit was proposed. The proposed operating procedure was studied experimentally using a one-machine infinite bus system with parallel transmission line including 3-phase SCFCL unit. All the SCFCLs for three phases started to limit the fault current at the 3-phase-short fault and recovered to the waiting mode by being short-circuited successfully. The fault line current continues to flow throughout the operation. It is pointed that the proposed operating procedure is better for the transient stability of the system than the conventional one is.

\section{REFERENCES}

[1] H. J. Boenig and D. A. Paice, "Fault current limiter using a superconducting coil," IEEE Trans. Magn., vol. 19, no. 3, pp. 1051-1053, May 1983. 
[2] T. Hara et al., "Development of a new $6.6 \mathrm{kV} / 1500 \mathrm{~A}$ class superconducting fault current limiter for electric power systems," IEEE Trans. Power Delivery, vol. 8, no. 1, pp. 182-192, Jan. 1993.

[3] E. Leung et al., "Design \& development of a $15 \mathrm{kV}$, 20kA HTS fault current limiter," IEEE Trans. Appl. Superconduct., vol. 10, no. 1, pp. 832-835, Mar. 2000.

[4] B. Gromoll et al., "Resistive fault current limiters with YBCO films-100kVA functional model," IEEE Trans. Appl. Superconduct., vol. 9, no. 2, pp. 656-659, June 1999.

[5] K. Fujikawa et al., "Experimental study on superconducting fault current limiter with adjustable trigger current level," in Proc. 15th Int. Conf. MT-15, Sept. 1998, pp. 571-574.
[6] Y. Shirai et al., "Study on recovery time of a superconducting fault current limiter with adjustable trigger current level," IEEE Trans. Appl. Superconduct., vol. 11, no. 1, pp. 2086-2089, Mar. 2001.

[7] H. Hatta et al., "Experimental study on sudden-short-circuit characteristic of synchronous generator with SCFCL," IEEE Trans. Appl. Superconduct., vol. 11, no. 1, pp. 2343-2346, Mar. 2001.

[8] K. Fujikawa et al., "Experimental study on adjustability of super-conducting fault current limiter with adjustable trigger current level," IEEE Trans. Appl. Superconduct., vol. 9, no. 2, pp. 1351-1354, June 1999. 